Article

\title{
Hermite-Hadamard-Type Inequalities for Convex Functions via the Fractional Integrals with Exponential Kernel
}

\author{
Xia Wu ${ }^{1}$, JinRong Wang ${ }^{2,3, *(1)}$ and Jialu Zhang ${ }^{1}$ \\ 1 School of Mathematics and Finance, Xiangnan University, Chenzhou 411105, China; \\ 230169714@seu.edu.cn (X.W.); zhangj1@xnu.edu.cn (J.Z.) \\ 2 Department of Mathematics, Guizhou University, Guiyang 550025, China \\ 3 School of Mathematical Sciences, Qufu Normal University, Qufu 273165, China \\ * Correspondence: jrwang@gzu.edu.cn
}

Received: 24 August 2019; Accepted: 9 September 2019; Published: 12 September 2019

\begin{abstract}
In this paper, we establish three fundamental integral identities by the first- and second-order derivatives for a given function via the fractional integrals with exponential kernel. With the help of these new fractional integral identities, we introduce a few interesting Hermite-Hadamard-type inequalities involving left-sided and right-sided fractional integrals with exponential kernels for convex functions. Finally, some applications to special means of real number are presented.
\end{abstract}

Keywords: convex functions, Hermite-Hadamard-type inequalities, fractional integrals, exponential kernel

MSC: 26A33

\section{Introduction}

Let $h:[a, b] \subset \Re \rightarrow \Re$ be a convex function. Then, $h$ meets the following classic Hermite-Hadamard inequality (see [1])

$$
h\left(\frac{a+b}{2}\right) \leq \frac{1}{b-a} \int_{a}^{b} h(s) d s \leq \frac{h(a)+h(b)}{2} .
$$

If $h$ is a concave function, the inequalities in (1) are presented in the negative direction. The Hermite-Hadamard inequality provides us the estimates for the integral average of a continuous convex function on a compact interval.

For the latest results on generalizing, improving, and extending this classical Hermite-Hadamard inequality, one can see [2-9] and the references therein.

In [10], Dragomir and Agarwal proved the following result connected with the right part of (1). In [11], Alomart also elicited the similar result for functions whose second derivatives absolute values are convex.

Lemma 1 (see [10], Theorem 2.2). Assuming $h:[a, b] \subseteq \Re \rightarrow \Re$ is a differentiable function, $h^{\prime} \in L[a, b]$ and $\left|h^{\prime \prime}\right|$ is convex on $[a, b]$. Then, the bellow inequality holds:

$$
\left|\frac{h(a)+h(b)}{2}-\frac{1}{b-a} \int_{a}^{b} h(s) d s\right| \leq \frac{(b-a)}{8}\left(\left|h^{\prime}(a)\right|+\left|h^{\prime}(b)\right|\right) .
$$


Lemma 2 (see [11], Theorem 3). Assuming $h:[a, b] \subseteq \Re \rightarrow \Re$ is a twice differentiable function, $h^{\prime \prime} \in L[a, b]$ and $\left|h^{\prime \prime}\right|$ is convex on $[a, b]$. Then, the following inequality holds

$$
\left|\frac{h(a)+h(b)}{2}-\frac{1}{b-a} \int_{a}^{b} h(s) d s\right| \leq \frac{(b-a)^{2}}{24}\left(\left|h^{\prime \prime}(a)\right|+\left|h^{\prime \prime}(b)\right|\right) .
$$

Now, fractional calculus has turned into an enchanting field of mathematics. Many extensive investigations have been carried out in this area. Due to the wide applications of Hermite-Hadamard inequalities and fractional integrals, many researchers have extended their research to Hermite-Hadamard inequalities involving fractional integrals rather than integer integrals, see [12-22]. Sarikaya et al. [12] have deduced an amusing inequality of Hermite-Hadamard-type involving fractional integrals in the place of ordinary integrals. This research fascinates many researchers to consider this respect. As a result, some new integral inequalities by the approach of fractional calculus have been obtained in the literature until now. In addition, Ahmad et al. [16] gave the new fractional integral operators with an exponential kernel and proved similar inequalities.

Definition 1 (see [16], Definition 2). Let $h \in L[a, b]$. The fractional left-side integral $\mathcal{J}_{a^{+}}^{\alpha} h$ and right-side integral $\mathcal{J}_{b^{-}}^{\alpha}$ of order $\alpha \in(0,1)$ are, respectively, defined by

$$
\mathcal{J}_{a^{+}}^{\alpha} h(x)=\frac{1}{\alpha} \int_{a}^{x} e^{-\frac{1-\alpha}{\alpha}(x-s)} h(s) d s, x>a,
$$

and

$$
\mathcal{J}_{b^{-}}^{\alpha} h(x)=\frac{1}{\alpha} \int_{x}^{b} e^{-\frac{1-\alpha}{\alpha}(x-s)} h(s) d s, \quad x<b .
$$

Lemma 3 (see [16], Theorem 1). Let $h:[a, b] \rightarrow \Re$ be a positive convex function with $0 \leq a<b$ and $h \in L[a, b]$. The following inequality for fractional integrals (4) and (5) holds:

$$
h\left(\frac{a+b}{2}\right) \leq \frac{1-\alpha}{2\left(1-e^{-\rho}\right)}\left[\mathcal{J}_{a^{+}}^{\alpha} h(b)+\mathcal{J}_{b^{-}}^{\alpha} h(a)\right] \leq \frac{h(a)+h(b)}{2} .
$$

Remark 1. In (6), note that

$$
\rho=\frac{1-\alpha}{\alpha}(b-a)
$$

In addition, Ahmad et al. [16] derived the bound estimate of the difference between the mean value of the endpoints and the average of the fractional integrals.

Lemma 4 (see [16], Theorem 3). Assuming $h:[a, b] \subseteq \Re \rightarrow \Re$ is differentiable, $h^{\prime} \in L[a, b]$ and $\left|h^{\prime}\right|$ are convex on $[a, b]$. Then, the following inequality holds:

$$
\left|Q_{m r}\right| \leq \frac{(b-a)}{2 \rho} \tanh \left(\frac{\rho}{4}\right)\left(\left|h^{\prime}(a)\right|+\left|h^{\prime}(b)\right|\right),
$$

where

$$
Q_{m r}:=\frac{h(a)+h(b)}{2}-\frac{1-\alpha}{2\left(1-e^{-\rho}\right)}\left[\mathcal{J}_{a^{+}}^{\alpha} h(b)+\mathcal{J}_{b^{-}}^{\alpha} h(a)\right]
$$

denotes the bound estimate of the difference between the mean value of the endpoints and the average of the fractional integrals. 
However, the bound for the left of the Hermite-Hadamard inequality (6) has not been studied. It will be interesting to find

$$
\left|Q_{m l}\right| \leq \text { what? }
$$

Here,

$$
Q_{m l}:=\frac{1-\alpha}{2\left(1-e^{-\rho}\right)}\left[\mathcal{J}_{a^{+}}^{\alpha} h(b)+\mathcal{J}_{b^{-}}^{\alpha} h(a)\right]-h\left(\frac{a+b}{2}\right)
$$

denotes the bound estimate of the difference between the value of the midpoint and the average of the fractional integrals.

Furthermore, if $\left|h^{\prime \prime}\right|$ is convex, it is natural to study the right- and left-type Hermite-Hadamard inequality via the fractional integral with an exponential kernel similar to Lemma 2, i.e., we want to find the constants $\rho_{1}$ and $\rho_{2}$ satisfying the following inequities:

$$
\left|Q_{m r}\right| \leq \rho_{1} \cdot\left(\left|h^{\prime \prime}(a)\right|+\left|h^{\prime \prime}(b)\right|\right),
$$

and

$$
\left|Q_{m l}\right| \leq \rho_{2} \cdot\left(\left|h^{\prime \prime}(a)\right|+\left|h^{\prime \prime}(b)\right|\right)
$$

Motivated by $[12,15,16]$, we will demonstrate three new fractional-type integral identities and set up their corresponding Hermite-Hadamard-type inequalities involving left-sided and right-sided fractional integrals for convex functions, respectively.

\section{New Fractional Integral Identity and Hermite-Hadamard-Type Inequality for First Order Derivative}

We firstly prove the following lemma in order to attest the following result.

Lemma 5. Assuming $h:[a, b] \subseteq \Re \rightarrow \Re$ is a differentiable mapping and $h^{\prime} \in L[a, b]$. Then, the following equality for the fractional integrals (4) and (5) holds:

$$
\begin{aligned}
Q_{m l}= & \frac{b-a}{2} \int_{0}^{1} k h^{\prime}(s a+(1-s) b) d s \\
& -\frac{b-a}{2\left(1-e^{-\rho}\right)}\left[\int_{0}^{1} e^{-\rho s} h^{\prime}(s a+(1-s) b) d s-\int_{0}^{1} e^{-\rho(1-s)} h^{\prime}(s a+(1-s) b) d s\right],
\end{aligned}
$$

where

$$
k=\left\{\begin{array}{r}
1,0 \leq s<\frac{1}{2} \\
-1, \frac{1}{2} \leq s \leq 1
\end{array}\right.
$$

Proof. Define

$$
\begin{aligned}
V & :=\int_{0}^{1} e^{-\rho s} h^{\prime}(s a+(1-s) b) d s-\int_{0}^{1} e^{-\rho(1-s)} h^{\prime}(s a+(1-s) b) d s \\
& =V_{1}-V_{2}
\end{aligned}
$$

where

$$
\begin{aligned}
& V_{1}=\int_{0}^{1} e^{-\rho s} h^{\prime}(s a+(1-s) b) d s \\
& V_{2}=\int_{0}^{1} e^{-\rho(1-s)} h^{\prime}(s a+(1-s) b) d s
\end{aligned}
$$


Integrating by parts, one has

$$
\begin{aligned}
V_{1} & =\int_{0}^{1} e^{-\rho s} h^{\prime}(s a+(1-s) b) d s \\
& =\frac{1}{a-b} \int_{0}^{1} e^{-\rho s} d(h(s a+(1-s) b)) \\
& =\frac{1}{a-b}\left[\left.e^{-\rho s} f(s a+(1-s) b)\right|_{0} ^{1}-\int_{0}^{1} h(s a+(1-s) b) d\left(e^{-\rho s}\right)\right] \\
& =\frac{1}{a-b}\left[e^{-\rho} h(a)-h(b)+\rho \int_{0}^{1} h(s a+(1-s) b) e^{-\rho s} d s\right] \\
& =\frac{e^{-\rho} h(a)-h(b)}{a-b}+\frac{\rho}{(a-b)^{2}} \int_{b}^{a} h(x) e^{-\rho \frac{x-b}{a-b}} d x \\
& =\frac{e^{-\rho} h(a)-h(b)}{a-b}-\frac{\rho}{(a-b)^{2}} \int_{a}^{b} e^{-\frac{1-\alpha}{\alpha}(b-x)} h(x) d x \\
& =\frac{e^{-\rho} h(a)-h(b)}{a-b}-\frac{\rho}{(a-b)^{2}} \alpha \mathcal{J}_{a^{+}}^{\alpha} h(b) \\
& =\frac{e^{-\rho} h(a)-h(b)}{a-b}-\frac{1-\alpha}{(b-a)} \mathcal{J}_{a^{+}}^{\alpha} h(b),
\end{aligned}
$$

and

$$
\begin{aligned}
V_{2} & =\int_{0}^{1} e^{-\rho(1-s)} h^{\prime}(s a+(1-s) b) d s \\
& =\frac{1}{a-b} \int_{0}^{1} e^{-\rho(1-s)} d(h(s a+(1-s) b)) \\
& =\frac{1}{a-b}\left[\left.e^{-\rho(1-s)} h(s a+(1-s) b)\right|_{0} ^{1}-\int_{0}^{1} h(s a+(1-s) b) d\left(e^{-\rho(1-s)}\right)\right] \\
& =\frac{1}{a-b}\left[h(a)-e^{-\rho} h(b)-\rho \int_{0}^{1} h(s a+(1-s) b) e^{-\rho(1-s)} d s\right] \\
& =\frac{h(a)-e^{-\rho} h(b)}{a-b}-\frac{\rho}{(a-b)^{2}} \int_{b}^{a} h(x) e^{-\rho \frac{a-x}{a-b}} d x \\
& =\frac{h(a)-e^{-\rho} h(b)}{a-b}+\frac{\rho}{(a-b)^{2}} \int_{a}^{b} e^{-\frac{1-\alpha}{\alpha}(x-a)} h(x) d x \\
& =\frac{h(a)-e^{-\rho} h(b)}{a-b}+\frac{\rho}{(a-b)^{2}} \alpha \mathcal{J}_{b^{-}}^{\alpha} h(a) \\
& =\frac{h(a)-e^{-\rho} h(b)}{a-b}+\frac{1-\alpha}{(b-a)} \mathcal{J}_{b^{-}}^{\alpha} h(a) .
\end{aligned}
$$

Substituting (9) and (10) into (8), we get that

$$
\begin{aligned}
V & =V_{1}-V_{2} \\
& =\frac{\left(1-e^{-\rho}\right)(h(a)+h(b))}{b-a}-\frac{1-\alpha}{(b-a)}\left[\mathcal{J}_{a^{+}}^{\alpha} h(b)+\mathcal{J}_{b^{-}}^{\alpha} h(a)\right] .
\end{aligned}
$$


Note

$$
\begin{aligned}
& \frac{b-a}{2} \int_{0}^{1} k h^{\prime}(s a+(1-s) b) d t \\
= & \frac{b-a}{2} \int_{0}^{\frac{1}{2}} h^{\prime}(s a+(1-s) b) d s-\frac{b-a}{2} \int_{\frac{1}{2}}^{1} h^{\prime}(s a+(1-s) b) d s \\
= & \frac{f(b)-h\left(\frac{a+b}{2}\right)}{2}+\frac{h(a)-h\left(\frac{a+b}{2}\right)}{2} \\
= & \frac{h(a)+h(b)}{2}-h\left(\frac{a+b}{2}\right) .
\end{aligned}
$$

Substituting (12) and (11) into the right-side of (7), we obtain the left of (7). This testifies the proof.

Then, we can declare the first theorem including Hermite-Hadmard-type inequality.

Theorem 1. If $h:[a, b] \subseteq \Re \rightarrow \Re$ is differentiable, $\left|h^{\prime}\right|$ is convex on $[a, b]$, and $h^{\prime} \in L[a, b]$, then the following inequality about the fractional integrals (4) and (5) holds:

$$
\left|Q_{m l}\right| \leq \frac{b-a}{2}\left[\frac{1}{2}-\frac{\tanh \left(\frac{\rho}{4}\right)}{\rho}\right]\left(\left|h^{\prime}(a)\right|+\left|h^{\prime}(b)\right|\right) .
$$

Proof. Using Lemma 5, convexity of $\left|h^{\prime}\right|$, and $e^{-\rho s} \geq e^{-\rho}$ and $e^{-\rho(1-s)} \geq e^{-\rho}$ for any $s \in[0,1]$, we obtain

$$
\begin{aligned}
& \left|Q_{m l}\right|=\mid \frac{b-a}{2} \int_{0}^{1} k h^{\prime}(s a+(1-s) b) d s \\
& -\frac{b-a}{2\left(1-e^{-\rho}\right)}\left[\int_{0}^{1} e^{-\rho s} h^{\prime}(s a+(1-s) b) d s-\int_{0}^{1} e^{-\rho(1-s)} h^{\prime}(s a+(1-s) b) d s\right] \mid \\
& =\frac{b-a}{2\left(1-e^{-\rho}\right)} \mid \int_{0}^{\frac{1}{2}}\left[\left(1-e^{-\rho}\right)-e^{-\rho s}+e^{-\rho(1-s)}\right] h^{\prime}(s a+(1-s) b) d s \\
& -\int_{\frac{1}{2}}^{1}\left[\left(1-e^{-\rho}\right)-e^{-\rho(1-s)}+e^{-\rho s}\right] h^{\prime}(s a+(1-s) b) d s \mid \\
& \leq \frac{b-a}{2\left(1-e^{-\rho}\right)}\left[\int_{0}^{\frac{1}{2}}\left(1-e^{-\rho}-e^{-\rho s}+e^{-\rho(1-s)}\right)\left(s\left|h^{\prime}(a)\right|+(1-s)\left|h^{\prime}(b)\right|\right) d s\right. \\
& \left.+\int_{\frac{1}{2}}^{1}\left(1-e^{-\rho}-e^{-\rho(1-s)}+e^{-\rho s}\right)\left(s\left|h^{\prime}(a)\right|+(1-s)\left|h^{\prime}(b)\right|\right) d s\right] \\
& =\frac{b-a}{2\left(1-e^{-\rho}\right)}\left[\int_{0}^{\frac{1}{2}}\left(1-e^{-\rho}-e^{-\rho s}+e^{-\rho(1-s)}\right)\left(s\left|h^{\prime}(a)\right|+(1-s)\left|h^{\prime}(b)\right|\right) d s\right. \\
& \left.+\int_{0}^{\frac{1}{2}}\left(1-e^{-\rho}-e^{-\rho s}+e^{-\rho(1-s)}\right)\left((1-s)\left|h^{\prime}(a)\right|+s\left|h^{\prime}(b)\right|\right) d s\right] \\
& =\frac{b-a}{2\left(1-e^{-\rho}\right)} \int_{0}^{\frac{1}{2}}\left(1-e^{-\rho}-e^{-\rho s}+e^{-\rho(1-s)}\right)\left(\left|h^{\prime}(a)\right|+\left|h^{\prime}(b)\right|\right) d s \\
& =\frac{b-a}{2\left(1-e^{-\rho}\right)}\left[\frac{1-e^{-\rho}}{2}-\frac{1}{\rho}\left(1-e^{-\frac{\rho}{2}}\right)^{2}\right]\left(\left|h^{\prime}(a)\right|+\left|h^{\prime}(b)\right|\right) \\
& =\frac{b-a}{2}\left[\frac{1}{2}-\frac{\tanh \left(\frac{\rho}{4}\right)}{\rho}\right]\left(\left|h^{\prime}(a)\right|+\left|h^{\prime}(b)\right|\right) \text {. }
\end{aligned}
$$

The proof is completed. 


\section{New Fractional Integral Identity and Hermite-Hadamard-Type Inequality for Second Order Derivative}

In [16] Lemma 4, Ahmad et al. gave the equality

$$
Q_{m r}=\frac{(b-a)}{2\left(1-e^{-\rho}\right)}\left[\int_{0}^{1} e^{-\rho s} h^{\prime}(s a+(1-s) b) d s-\int_{0}^{1} e^{-\rho(1-s)} h^{\prime}(s a+(1-s) b) d s\right] .
$$

By (14), we will prove the Hermite-Hadamard-type inequality of the order derivatives via the fractional integrals with an exponential kernel for convex functions. Before we prove our main results in this section, we give the following lemmas.

Lemma 6. Assuming $h:[a, b] \rightarrow \Re$ is a twice differentiable function. If $h^{\prime \prime} \in L[a, b]$, then the following equality for fractional integrals holds:

$$
Q_{m r}=\frac{(b-a)^{2}}{2 \rho\left(1-e^{-\rho}\right)} \int_{0}^{1}\left(1+e^{-\rho}-e^{-\rho s}-e^{-\rho(1-s)}\right) h^{\prime \prime}(s a+(1-s) b) d s .
$$

Proof. By using equality (14), we note

$$
\begin{aligned}
K_{1} & =\int_{0}^{1} e^{-\rho s} h^{\prime}(s a+(1-s) b) d s=-\frac{1}{\rho} \int_{0}^{1} h^{\prime}(s a+(1-s) b) d\left(e^{-\rho s}\right) \\
& =\frac{1}{\rho}\left[h^{\prime}(b)-e^{-\rho} h^{\prime}(a)+(a-b) \int_{0}^{1} e^{-\rho s} h^{\prime \prime}(s a+(1-s) b) d s\right]
\end{aligned}
$$

and

$$
\begin{aligned}
K_{2} & =\int_{0}^{1} e^{-\rho(1-s)} h^{\prime}(s a+(1-s) b) d s=\frac{1}{\rho} \int_{0}^{1} h^{\prime}(s a+(1-s) b) d\left(e^{-\rho(1-s)}\right) \\
& =\frac{1}{\rho}\left[h^{\prime}(a)-e^{-\rho} h^{\prime}(b)-(a-b) \int_{0}^{1} e^{-\rho(1-s)} h^{\prime \prime}(s a+(1-s) b) d s\right] .
\end{aligned}
$$

Inserting the values of $K_{1}$ and $K_{2}$ in (14), we obtain

$$
\begin{aligned}
& \frac{h(a)+h(b)}{2}-\frac{1-\alpha}{2\left(1-e^{-\rho}\right)}\left[\mathcal{J}_{a^{+}}^{\alpha} h(b)+\mathcal{J}_{b^{-}}^{\alpha} h(a)\right] \\
= & \frac{b-a}{2 \rho\left(1-e^{-\rho}\right)}\left[\left(1+e^{-\rho}\right)\left(h^{\prime}(b)-h^{\prime}(a)\right)\right. \\
& \left.-(b-a) \int_{0}^{1}\left(e^{-\rho s}+e^{-\rho(1-s)}\right) h^{\prime \prime}(s a+(1-s) b) d s\right] \\
= & \frac{b-a}{2 \rho\left(1-e^{-\rho}\right)}\left[\left(1+e^{-\rho}\right)(b-a) \int_{0}^{1} h^{\prime \prime}(s a+(1-s) b) d s\right. \\
& \left.-(b-a) \int_{0}^{1}\left(e^{-\rho s}+e^{-\rho(1-s)}\right) h^{\prime \prime}(s a+(1-s) b) d s\right] \\
= & \frac{(b-a)^{2}}{2 \rho\left(1-e^{-\rho}\right)} \int_{0}^{1}\left(1+e^{-\rho}-e^{-\rho s}-e^{-\rho(1-s)}\right) h^{\prime \prime}(s a+(1-s) b) d s .
\end{aligned}
$$

This completes the proof.

Lemma 7. Assuming $h:[a, b] \rightarrow \Re$ is a twice differentiable function. If $h^{\prime \prime} \in L[a, b]$, then the following equality for fractional integrals holds:

$$
Q_{m l}=\frac{(b-a)^{2}}{2} \int_{0}^{1} m(s) h^{\prime \prime}(s a+(1-s) b) d s,
$$


where

$$
m(s)=\left\{\begin{array}{l}
s-\frac{1+e^{-\rho}-e^{-\rho s}-e^{-\rho(1-s)}}{\rho\left(1-e^{-\rho}\right)}, \quad 0 \leq s<\frac{1}{2}, \\
(1-s)-\frac{1+e^{-\rho}-e^{-\rho s}-e^{-\rho(1-s)}}{\rho\left(1-e^{-\rho}\right)}, \quad \frac{1}{2} \leq s<1 .
\end{array}\right.
$$

Proof. By using the proof of the Lemma 5, we can get

$$
\begin{aligned}
Q_{m l}= & \frac{b-a}{2} \int_{0}^{1} k h^{\prime}(s a+(1-s) b) d s \\
& -\frac{b-a}{2\left(1-e^{-\rho}\right)}\left[\int_{0}^{1} e^{-\rho s} h^{\prime}(s a+(1-s) b) d s-\int_{0}^{1} e^{-\rho(1-s)} h^{\prime}(s a+(1-s) b) d s\right] .
\end{aligned}
$$

Thus,

$$
\begin{aligned}
& \frac{b-a}{2} \int_{0}^{1} k h^{\prime}(s a+(1-s) b) d s \\
= & \frac{b-a}{2} \int_{0}^{\frac{1}{2}} h^{\prime}(s a+(1-s) b) d s-\frac{b-a}{2} \int_{\frac{1}{2}}^{1} h^{\prime}(s a+(1-s) b) d s \\
= & \frac{b-a}{2}\left[\left.s h^{\prime}(s a+(1-s) b)\right|_{0} ^{\frac{1}{2}}-(a-b) \int_{0}^{\frac{1}{2}} s h^{\prime \prime}(s a+(1-s) b) d s\right] \\
& -\frac{b-a}{2}\left[\left.s h^{\prime}(s a+(1-s) b)\right|_{\frac{1}{2}} ^{1}-(a-b) \int_{\frac{1}{2}}^{1} s h^{\prime \prime}(s a+(1-s) b) d s\right] \\
= & \frac{b-a}{2}\left[\frac{1}{2} h^{\prime}\left(\frac{a+b}{2}\right)-(a-b) \int_{0}^{\frac{1}{2}} s h^{\prime \prime}(s a+(1-s) b) d s\right] \\
& -\frac{b-a}{2}\left[h^{\prime}(a)-\frac{1}{2} h^{\prime}\left(\frac{a+b}{2}\right)-(a-b) \int_{\frac{1}{2}}^{1} s h^{\prime \prime}(s a+(1-s) b) d s\right] \\
= & \frac{b-a}{2}\left[h^{\prime}\left(\frac{a+b}{2}\right)-h^{\prime}(a)\right] \\
& +\frac{(b-a)^{2}}{2} \int_{0}^{\frac{1}{2}} s h^{\prime \prime}(s a+(1-s) b) d s-\frac{(b-a)^{2}}{2} \int_{\frac{1}{2}}^{1} s h^{\prime \prime}(s a+(1-s) b) d s \\
= & \frac{(b-a)^{2}}{2} \int_{\frac{1}{2}}^{1} h^{\prime \prime}(s a+(1-s) b) d s \\
& +\frac{(b-a)^{2}}{2} \int_{0}^{\frac{1}{2}} s h^{\prime \prime}(s a+(1-s) b) d s-\frac{(b-a)^{2}}{2} \int_{\frac{1}{2}}^{1} s h^{\prime \prime}(s a+(1-s) b) d s \\
= & \frac{(b-a)^{2}}{2} \int_{0}^{\frac{1}{2}} s h^{\prime \prime}(s a+(1-s) b) d s+\frac{(b-a)^{2}}{2} \int_{\frac{1}{2}}^{1}(1-s) h^{\prime \prime}(s a+(1-s) b) d s .
\end{aligned}
$$

Submitting (16), (17), and (21) to (20), we get (19). This completes the proof.

Now, we can prove our Hermite-Hadamard-type inequalities by the second order derivatives.

Theorem 2. Assuming $h:[a, b] \rightarrow \Re$ is a twice differentiable function. If $h^{\prime \prime} \in L[a, b]$ and $\left|h^{\prime \prime}\right|$ is convex on $[a, b]$, the following inequality for fractional integrals with exponential kernel holds:

$$
\left|Q_{m r}\right| \leq \frac{(b-a)^{2}}{2 \rho\left(1-e^{-\rho}\right)}\left(\frac{1+e^{-\rho}}{2}-\frac{1-e^{-\rho}}{\rho}\right)\left(\left|h^{\prime \prime}(a)\right|+\left|h^{\prime \prime}(b)\right|\right) .
$$


Proof. Note that

$$
\begin{aligned}
& \int_{0}^{1}\left(1+e^{-\rho}-e^{-\rho s}-e^{-\rho(1-s)}\right) s d s \\
= & \left(1+e^{-\rho}\right) \int_{0}^{1} s d s-\int_{0}^{1} s e^{-\rho s} d s-\int_{0}^{1} s e^{-\rho(1-s)} d s \\
= & \frac{1+e^{-\rho}}{2}+\frac{1}{\rho}\left(e^{-\rho}-\frac{1-e^{-\rho}}{\rho}\right)-\frac{1}{\rho}\left(1-\frac{1-e^{-\rho}}{\rho}\right) \\
= & \frac{1+e^{-\rho}}{2}-\frac{1-e^{-\rho}}{\rho},
\end{aligned}
$$

and

$$
\begin{aligned}
& \int_{0}^{1}\left(1+e^{-\rho}-e^{-\rho s}-e^{-\rho(1-s)}\right)(1-s) d s \\
= & \int_{0}^{1}\left(1+e^{-\rho}-e^{-\rho s}-e^{-\rho(1-s)}\right) d s-\int_{0}^{1} s\left(1+e^{-\rho}-e^{-\rho s}-e^{-\rho(1-s)}\right) d s \\
= & \left.\left(1+e^{-\rho}\right)\right|_{0} ^{1}+\left.\frac{e^{-\rho s}}{\rho}\right|_{0} ^{1}-\left.\frac{e^{-\rho(1-s)}}{\rho}\right|_{0} ^{1}-\left(\frac{1+e^{-\rho}}{2}-\frac{1-e^{-\rho}}{\rho}\right) \\
= & \frac{1+e^{-\rho}}{2}-\frac{1-e^{-\rho}}{\rho} .
\end{aligned}
$$

According to (15), (23), (24), and the convex of $\left|h^{\prime \prime}\right|$, we can get

$$
\begin{aligned}
\left|Q_{m r}\right| & =\left|\frac{(b-a)^{2}}{2 \rho\left(1-e^{-\rho}\right)} \int_{0}^{1}\left(1+e^{-\rho}-e^{-\rho s}-e^{-\rho(1-s)}\right) h^{\prime \prime}(s a+(1-s) b) d s\right| \\
& \leq \frac{(b-a)^{2}}{2 \rho\left(1-e^{-\rho}\right)} \int_{0}^{1}\left|1+e^{-\rho}-e^{-\rho s}-e^{-\rho(1-s)}\right|\left|h^{\prime \prime}(s a+(1-s) b)\right| d s \\
& \leq \frac{(b-a)^{2}}{2 \rho\left(1-e^{-\rho}\right)} \int_{0}^{1}\left(1+e^{-\rho}-e^{-\rho s}-e^{-\rho(1-s)}\right)\left(s\left|h^{\prime \prime}(a)\right|+(1-s)\left|h^{\prime \prime}(b)\right|\right) d s \\
& =\frac{(b-a)^{2}}{2 \rho\left(1-e^{-\rho}\right)}\left(\frac{1+e^{-\rho}}{2}-\frac{1-e^{-\rho}}{\rho}\right)\left(\left|h^{\prime \prime}(a)\right|+\left|h^{\prime \prime}(b)\right|\right) .
\end{aligned}
$$

The proof is finished.

Remark 2. $\alpha \rightarrow 1$ in (22) of Theorem 2, then $\rho=\frac{1-\alpha}{\alpha}(b-a) \rightarrow 0$, one obtains

$$
\lim _{\rho \rightarrow 0} \frac{1-\alpha}{2\left(1-e^{-\rho}\right)}=\lim _{x \rightarrow 1} \frac{1-\frac{b-a}{b-a-\ln x}}{2(1-x)}=\lim _{x \rightarrow 1} \frac{-\ln x}{2(1-x)(b-a-\ln x)}=\frac{1}{2(b-a)},
$$

and

$$
\lim _{\rho \rightarrow 0} \frac{1}{\rho\left(1-e^{-\rho}\right)}\left(\frac{1+e^{-\rho}}{2}-\frac{1-e^{-\rho}}{\rho}\right)=\lim _{\rho \rightarrow 0} \frac{\rho+\rho e^{-\rho}-2+2 e^{-\rho}}{2 \rho^{2}\left(1-e^{-\rho}\right)}=\frac{1}{12} .
$$

So (22) is transformed to

$$
\left|\frac{h(a)+h(b)}{2}-\frac{1}{b-a} \int_{a}^{b} h(s) d s\right| \leq \frac{(b-a)^{2}}{24}\left(\left|h^{\prime \prime}(a)\right|+\left|h^{\prime \prime}(b)\right|\right) .
$$

This result coincides the conclusion in [11], Theorem 3. 
Theorem 3. Assuming $h:[a, b] \rightarrow \Re$ is a twice differentiable function. If $h^{\prime \prime} \in L[a, b]$ and $\left|h^{\prime \prime}\right|$ is convex on $[a, b]$, then the following inequality for fractional integrals with exponential kernel holds:

$$
\left|Q_{m l}\right| \leq \frac{(b-a)^{2}}{2}\left[\frac{1}{8}+\frac{1+e^{-\rho}}{2 \rho\left(1-e^{-\rho}\right)}-\frac{1}{\rho^{2}}\right]\left(\left|h^{\prime \prime}(a)\right|+\left|h^{\prime \prime}(b)\right|\right) .
$$

Proof. According to Lemma 7 and the convex of $\left|h^{\prime \prime}\right|$, we can get

$$
\begin{aligned}
\left|Q_{m l}\right|= & \left|\frac{(b-a)^{2}}{2} \int_{0}^{1} m(s) h^{\prime \prime}(s a+(1-s) b) d s\right| \leq \frac{(b-a)^{2}}{2} \int_{0}^{1}|m(s)|\left|h^{\prime \prime}(s a+(1-s) b)\right| d s \\
\leq & \frac{(b-a)^{2}}{2} \int_{0}^{\frac{1}{2}}\left(s+\frac{1+e^{-\rho}-e^{-\rho s}-e^{-\rho(1-s)}}{\rho\left(1-e^{-\rho}\right)}\right)\left(s\left|h^{\prime \prime}(a)\right|+(1-s)\left|h^{\prime \prime}(b)\right|\right) d s \\
& +\frac{(b-a)^{2}}{2} \int_{\frac{1}{2}}^{1}\left(1-s+\frac{1+e^{-\rho}-e^{-\rho s}-e^{-\rho(1-s)}}{\rho\left(1-e^{-\rho}\right)}\right)\left(s\left|h^{\prime \prime}(a)\right|+(1-s)\left|h^{\prime \prime}(b)\right|\right) d s \\
= & \frac{(b-a)^{2}}{2}\left[\int_{0}^{\frac{1}{2}}\left(s^{2}\left|h^{\prime \prime}(a)\right|+t(1-t)\left|h^{\prime \prime}(b)\right|\right) d s+\int_{\frac{1}{2}}^{1}\left(s(1-s)\left|h^{\prime \prime}(a)\right| d s+(1-s)^{2}\left|h^{\prime \prime}(b)\right|\right) d s\right. \\
& \left.+\frac{1}{\rho\left(1-e^{-\rho}\right)} \int_{0}^{1}\left(1+e^{-\rho}-e^{-\rho s}-e^{-\rho(1-s)}\right)\left(s\left|h^{\prime \prime}(a)\right|+(1-s)\left|h^{\prime \prime}(b)\right|\right) d s\right] \\
= & \frac{(b-a)^{2}}{2}\left[\frac{1}{24}\left|h^{\prime \prime}(a)\right|+\frac{1}{12}\left|h^{\prime \prime}(b)\right|+\frac{1}{12}\left|h^{\prime \prime}(a)\right|+\frac{1}{24}\left|h^{\prime \prime}(b)\right|\right. \\
& \left.+\frac{1}{\rho\left(1-e^{-\rho}\right)}\left(\frac{1+e^{-\rho}}{2}-\frac{1-e^{-\rho}}{\rho}\right)\left(\left|h^{\prime \prime}(a)\right|+\left|h^{\prime \prime}(b)\right|\right)\right] \\
= & \frac{(b-a)^{2}}{2}\left[\frac{1}{8}+\frac{1+e^{-\rho}}{2 \rho\left(1-e^{-\rho}\right)}-\frac{1}{\rho^{2}}\right]\left(\left|h^{\prime \prime}(a)\right|+\left|h^{\prime \prime}(b)\right|\right) .
\end{aligned}
$$

This completes the proof.

Remark 3. Let $\alpha \rightarrow 1$ in (27), one has

$$
\left|\frac{1}{b-a} \int_{a}^{b} h(s) d s-h\left(\frac{a+b}{2}\right)\right| \leq \frac{5(b-a)^{2}}{48}\left(\left|h^{\prime \prime}(a)\right|+\left|h^{\prime \prime}(b)\right|\right) .
$$

\section{Application to Special Means}

Think on the following particular means [23] for $\forall p, q \in R, p \neq q$ as follows:

$$
\begin{aligned}
& \text { (i) } \quad H(p, q)=\frac{2}{\frac{1}{p}+\frac{1}{q}}, p, q \in R \backslash\{0\} ; \\
& \text { (ii) } \quad A(p, q)=\frac{p+q}{2}, p, q \in R ; \\
& \text { (iii) } \quad L(p, q)=\frac{q-p}{\ln |p|-\ln |q|},|p| \neq|q|, p q \neq 0 ; \\
& \text { (iv) } L_{m}(p, q)=\left[\frac{q^{m+1}-p^{m+1}}{(m+1)(q-p)}\right]^{\frac{1}{m}}, m \in Z \backslash\{-1,0\}, p, q \in R, p \neq q .
\end{aligned}
$$

Next, making use of the acquired results in Section 3, we give some applications to particular means of real number.

Proposition 1. Let $p, q \in \Re, p<q, p q>0$ and $m \in \mathbb{Z},|m| \geq 2$. Then,

$$
\left|L_{m}^{m}(p, q)-A^{m}(p, q)\right| \leq \frac{5}{24}(q-p)^{2}|m(m-1)| A\left(|p|^{m-2},|q|^{m-2}\right) .
$$


Proof. Applying Remark 3 for $h(x)=x^{m}$, we can get the conclusion immediately.

The upper bound is smaller than the result of Proposion 3.1 in [5] when $|q-p| \leq 1$ and $|p|,|q|>1$ obviously.

Proposition 2. Let $p, q \in \Re, p<q, p q>0$. Then,

$$
\left|L^{-1}(p, q)-A^{-1}(p, q)\right| \leq \frac{5}{12}(q-p)^{2} A\left(|p|^{-3},|q|^{-3}\right) .
$$

Proof. The inference follows from Remark 3 used for $h(x)=\frac{1}{x}$.

Proposition 3. Let $p, q \in \Re, p<q, p q>0$ and $m \in \mathbb{Z},|m| \geq 2$. Then, we have

$$
\left|L_{m}^{m}\left(q^{-1}, p^{-1}\right)-H^{-m}(q, p)\right| \leq \frac{5}{24}\left(\frac{1}{p}-\frac{1}{q}\right)^{2}|m(m-1)| H^{-1}\left(|p|^{m-2},|q|^{m-2}\right),
$$

and

$$
\left|L^{-1}(p, q)-H(p, q)\right| \leq \frac{5}{12}(q-p)^{2} H^{-1}\left(|p|^{3},|q|^{3}\right) .
$$

Proof. Doing he replacement $q^{-1} \rightarrow p, p^{-1} \rightarrow q$ in the inequalities (28) and (29), we can obtain the required inequalities (30) and (31), respectively. Here, we have observed $A^{-1}\left(p^{-1}, q^{-1}\right)=H(p, q)=$ $2 /\left(\frac{1}{p}+\frac{1}{q}\right), q^{-1}<p^{-1}$.

At last, we will present an application to a midpoint formula. In [23], let $w$ be a division $p=s_{0}<s_{1} \cdots<s_{m-1}<s_{m}=q$ of the interval $[p, q]$ and inspect the quadrature formula

$$
\int_{p}^{q} h(s) d s=T(h, w)+E(h, w)
$$

where

$$
T(h, w)=\sum_{i=0}^{m-1} h\left(\frac{s_{i}+s_{i+1}}{2}\right)\left(s_{i+1}-s_{i}\right)
$$

is the midpoint version and $E(h, w)$ refers to the approximation error. Here, we deduce the error estimate for the midpoint formula.

Proposition 4. Let $h:[p, q] \rightarrow \Re$ be a twice differentiable mapping on $(p, q)$ with $p<q$. If $h^{\prime \prime} \in L[a, b]$ and $\left|h^{\prime \prime}\right|$ is convex on $[p, q]$, then in (32), for every division $w$ of $[p, q]$, the following inequality holds:

$$
|E(f, w)| \leq \frac{5}{48} \sum_{i=0}^{m-1}\left(s_{i+1}-s_{i}\right)^{3}\left(\left|h^{\prime \prime}\left(s_{i}\right)\right|+\left|h^{\prime \prime}\left(s_{i+1}\right)\right|\right) .
$$

Proof. Applying Remark 3 on subinterval $\left[s_{i}, s_{i+1}\right](i=0,1, \cdots, m-1)$ of the division $w$, we derive

$$
\left|\int_{s_{i}}^{s_{i+1}} h(s) d s-h\left(\frac{s_{i}+s_{i+1}}{2}\right)\left(s_{i}-s_{i+1}\right)\right| \leq \frac{5}{48}\left(s_{i+1}-s_{i}\right)^{3}\left(\left|h^{\prime \prime}\left(s_{i}\right)\right|+\left|h^{\prime \prime}\left(s_{i+1}\right)\right|\right) .
$$


Summing over from 0 to $m-1$ and making use of the convexity of $\left|h^{\prime \prime}\right|$, we infer that

$$
\begin{aligned}
& \left|\int_{p}^{q} h(s) d s-T(h, w)\right|=\left|\sum_{i=0}^{m-1}\left[\int_{s_{i}}^{s_{i+1}} h(s) d s-f\left(\frac{s_{i}+s_{i+1}}{2}\right)\left(s_{i}-s_{i+1}\right)\right]\right| \\
\leq & \sum_{i=0}^{m-1}\left|\int_{s_{i}}^{s_{i+1}} h(s) d s-h\left(\frac{s_{i}+s_{i+1}}{2}\right)\left(s_{i}-s_{i+1}\right)\right| \\
\leq & \frac{5}{48} \sum_{i=0}^{m-1}\left(s_{i+1}-s_{i}\right)^{3}\left(\left|h^{\prime \prime}\left(s_{i}\right)\right|+\left|h^{\prime \prime}\left(s_{i+1}\right)\right|\right) .
\end{aligned}
$$

The proof is completed.

\section{Conclusions}

Based on the above interpretation, we acquire the bound estimates of the difference between the average of the fractional integrals with an exponential kernel and the mean values of the endpoints and the midpoint.

By comparing these bound estimates, we have obtained the following conclusions:

(i) With the first and second order derivatives of a given function, the Hermite-Hadamard-type inequalities involving left-sided and right-sided, the fractional integrals are different. The Hermite-Hadamard-type inequalities with the second order derivatives of a given function are more accurate.

(ii) With the same order derivatives of a given function, the Hermite-Hadamard-type inequalities involving different fractional integrals finally tend to be same when $\alpha \rightarrow 1$.

Author Contributions: Conceptualization and supervision, J.W.; Formal analysis and writing-original draft preparation, X.W., J.Z.; Writing-review and editing, X.W., J.W..

Funding: The authors thank the referees for their careful reading of the article and insightful comments. This work is partially supported by "Applied Mathematics" as a Key Construction Subject in the 12th Five-Year Plan of Hunan Province, Hunan Natural Science Foundation (2017J2241) and Hunan Social Science Foundation Subsidized Project (16YBA329).

Acknowledgments: The authors thank the referees for their careful reading of the article and insightful comments.

Conflicts of Interest: The authors declare no conflict of interest.

\section{References}

1. Mitrinović, D.S.; Lackovixcx, I.B. Hermite and convexity. Aequ. Math. 1985, 28, 229-232. [CrossRef]

2. Abramovich, S.; Barić, J.; Pexcxarixcx, J. Fejer and Hermite-Hadamard type inequalities for superquadratic functions. J. Math. Anal. Appl. 2008, 344, 1048-1056. [CrossRef]

3. Cal, J.; Carcamob, J.; Escauriaza, L. A general multidimensional Hermite-Hadamard type inequality. J. Math. Anal. Appl. 2009, 356, 659-663. [CrossRef]

4. Ödemir, M.E.; Avci, M.; Set, E. On some inequalities of Hermite-Hadamard type via m-convexity. Appl. Math. Lett. 2010, 23, 1065-1070. [CrossRef]

5. Xiao, Z.; Zhang, Z.; Wu, Y. On weighted Hermite-Hadamard inequalities. Appl. Math. Comput. 2011, 218, 1147-1152. [CrossRef]

6. Niculescu, C.P. The Hermite-Hadamard inequality for log-convex functions. Nonlinear Anal. TMA 2012, 75, 662-669. [CrossRef]

7. Dragomir, S.S. Inequalities of Hermite-Hadamard type for h-convex functions on linear spaces. Proyecc. J. Math. 2015, 34, 323-341. [CrossRef]

8. Muhammad, A.K.; Chu, Y.; Tahir, U.K.; Jamroz, K. Some new inequalities of Hermite-Hadamard type for s-convex functions with applications. Open Math. 2017, 15, 1414-1430.

9. Budak, H.; Sarikaya, M.Z. Journal of Mathematical Extension, Some new generalized Hermite-Hadamard inequalities for generalized convex functions and applications. Math. Ext. 2018, 12, 51-56. 
10. Dragomir, S.S.; Agarwal, R.P. Two inequalities for differentiable mappings and applications to special means of real numbers and to trapezoidal formula. Appl. Math. Lett. 1998, 11, 91-95. [CrossRef]

11. Alomari, M.; Darus, M.; Dragomir, S.S. New inequalities of Hermite-Hadamard type for functions whose second derivates absolute values are quasiconvex. RGMIA Res. Rep. Coll. 2010, 12. [CrossRef]

12. Sarikaya, M.Z.; Set, E.; Yaldiz, H.; Başak, N. Hermite-Hadamard's inequalities for fractional integrals and related fractional inequalities. Math. Comput. Modell. 2013, 57, 2403-2407. [CrossRef]

13. İŞcan, I.; Wu, S. Hermite-Hadamard type inequalities for harmonically convex functions via fractional integrals. Appl. Math. Comput. 2014, 238, 237-244. [CrossRef]

14. Jleli, M.; O'Regan, M.; Samet, B. On Hermite-Hadamard type inequalities via generalized fractional integrals. Turk. J. Math. 2016, 40, 1221-1230. [CrossRef]

15. Noor, M.A.; Noor, K.I.; Awan, M.U.; Khan, S. Fractional Hermite-Hadamard inequalities for some new classes of Godunova-Levin functions. Appl. Math. Inf. Sci. 2014, 8, 2865-2872. [CrossRef]

16. Ahmad, B.; Alsaedi, A.; Kirane, M.; Torebek, B.T. Hermite-Hadamard, Hermite-Hadamard-Fejér, Dragomir-Agarwal and Pachpatte type inequalities for convex functions via new fractional integrals. J. Comput. Appl. Math. 2019, 353, 120-129. [CrossRef]

17. Wang, J.; Zhu, C.; Zhou, Y. New generalized Hermite-Hadamard type inequalities and applications to specialmeans. J. Inequ. Appl. 2013, 325, 1-15.

18. Set, E.; Çelik, B. Generaliaed Fractional Hermite-Hadamard type inequalities for $m$-Convex and $(\alpha, m)$-Convex funciton. Commun. Fac. Sci. Univ. Ank. Ser. Math. Stat. 2018, 67, 333-344.

19. Chen, H.; Katugampola, U.N. Hermite-Hadamard and Hermite-Hadamard-Fejér type inequalities for generalized fractional integrals. J. Math. Anal. Appl. 2017, 446, 1274-1291. [CrossRef]

20. Set, E.; Noor, M.A.; Awan, M.U.; Gözpinar, A. Generalized Hermite-Hadamard type inequalities involving fractional integral operators. J. Inequ. Appl. 2017, 169, 1-10. [CrossRef]

21. Wang, J.; Li, X.; Zhou, Y. Hermite-Hadamard inequalities involving Riemann-Liouville fractional integrals via s-convex functions and applications to special means. Filomat 2016, 30, 1143-1150,. [CrossRef]

22. Liu, W.; Wen, W.; Park, J. Hermite-Hadamard type inequalities for MT-convex functions via classical integrals and fractional integrals. J. Nonlinear Sci. Appl. 2016, 9, 766-777. [CrossRef]

23. Pearce, C.E.M.; Pećarixcx, J. Inequalities for Differentiable Mappings with Application to Special Means and Quadrature Formula. Appl. Math. Lett. 2000, 13, 51-55. [CrossRef]

(C) 2019 by the authors. Licensee MDPI, Basel, Switzerland. This article is an open access article distributed under the terms and conditions of the Creative Commons Attribution (CC BY) license (http:/ / creativecommons.org/licenses/by/4.0/). 